\title{
Added Value of Assessing Adnexal Masses with Advanced MRI Techniques
}

\author{
I. Thomassin-Naggara, ${ }^{1,2,3}$ D. Balvay, ${ }^{1}$ A. Rockall, ${ }^{4}$ M. F. Carette, ${ }^{2,3}$ \\ M. Ballester, ${ }^{3,5}$ E. Darai, ${ }^{3,5}$ and M. Bazot ${ }^{2,3}$ \\ ${ }^{1}$ INSERM, UMR970, Team 2, Angiogenesis Imaging, 75005 Paris, France \\ ${ }^{2}$ AP-HP, Hôpital Tenon, Department of Radiology, 58 avenue Gambetta, 75020 Paris, France \\ ${ }^{3}$ Sorbonne University, UPMC Univ Paris 06, IUC, 75005 Paris, France \\ ${ }^{4}$ Imperial College London, ICTEM Building, Room 136, 1st Floor, Du Cane Road, London W12 0NN, UK \\ ${ }^{5}$ AP-HP, Hôpital Tenon, Department of Gynaecology and Obstetrics, 4 rue de la Chine, 75020 Paris, France
}

Correspondence should be addressed to I. Thomassin-Naggara; isabelle.thomassin@tnn.aphp.fr

Received 10 August 2014; Revised 23 November 2014; Accepted 7 December 2014

Academic Editor: Zhengchao Dong

Copyright (C) 2015 I. Thomassin-Naggara et al. This is an open access article distributed under the Creative Commons Attribution License, which permits unrestricted use, distribution, and reproduction in any medium, provided the original work is properly cited.

\begin{abstract}
This review will present the added value of perfusion and diffusion MR sequences to characterize adnexal masses. These two functional MR techniques are readily available in routine clinical practice. We will describe the acquisition parameters and a method of analysis to optimize their added value compared with conventional images. We will then propose a model of interpretation that combines the anatomical and morphological information from conventional MRI sequences with the functional information provided by perfusion and diffusion weighted sequences.
\end{abstract}

\section{Introduction}

The clinical suspicion of an adnexal mass is one of the most frequent indications for gynecologic imaging. In this setting, the first imaging technique is ultrasonography with Doppler [1-6]. A large majority of women will not need any other imaging technique for characterization of the adnexal lesion because the lesion either has typically benign features (such as anechoic cyst) or is overtly malignant (such as the presence of peritoneal implants) [7]. In the latter case, the extent of disease will then be determined with computed tomography (CT) which is the current standard of care for preoperative staging.

However, when an echoic area is discovered at ultrasound, the question remains whether or not this represents a solid soft tissue component consistent with tumour. If the sonographer detects blood flow within the echoic area, a solid component surely exists but many benign lesions may display a solid component [8]. If no blood flow is detected in the echoic portion, the question remains without answer as solid tissue may not display any blood flow on ultrasound [9]. Thus, when no typical signs of benignity or malignancy are present, the lesion remains indeterminate and a second line technique is needed with a frequency that depends on sonographer's experience $[10,11]$.

Pelvic magnetic resonance (MR) imaging has clearly been demonstrated to be the best imaging technique to characterize indeterminate or complex adnexal masses due to its excellent tissue contrast $[12,13]$. Firstly, the conventional sequences (T2, T1, and $\mathrm{T} 1$ with fat saturation) are evaluated. MR imaging is very accurate for the identification of endometriotic or fatty masses using these sequences whereas sonography can only suggest these diagnoses in some cases $[14,15]$. Moreover, conventional MR imaging readily distinguishes cystic from solid soft tissue components, as solid soft tissue enhances after gadolinium injection [16, 17]. Malignancy can only be suggested if there are enhancing solid components, such as thickened irregular septa, solid papillary projections, or a solid mass. These enhancing solid components are grouped under the name of "solid tissue" [18, 19]. 
TABLE 1: Acquisition parameters.

\begin{tabular}{|c|c|}
\hline Sequences & Parameters \\
\hline Sag T2 without FS & $\begin{array}{l}\text { (i) FOV: } 24 \mathrm{~cm} \\
\text { (ii) Thickness } \leq 6 \mathrm{~mm} \\
\text { (iii) }+/- \text { motion correction if available }\end{array}$ \\
\hline Ax T2 without FS & $\begin{array}{l}\text { (i) FOV: } 30 \mathrm{~cm} \text {, from kidney to symphysis. } \\
\text { (ii) Thickness: } 5 \mathrm{~mm} / 1.0 \text { spacing }\end{array}$ \\
\hline $\begin{array}{l}\text { Ax } \mathrm{T} 1 \\
\text { Ax or sag } \mathrm{T} 1 \mathrm{FS}\end{array}$ & $\begin{array}{l}\text { (i) Exact same location as Ax T2 without FS } \\
\text { (ii) FOV: } 30 \mathrm{~cm} \\
\text { (a) } 2 \mathrm{D} \text { thickness } 5 \mathrm{~mm} / 1.0 \text { spacing } \\
\text { (b) } 3 \mathrm{D} \text { reconstruction thickness } 3 \mathrm{~mm} / 0.0 \text { spacing }\end{array}$ \\
\hline Ax DWI & $\begin{array}{l}\text { (i) Exact same location as Ax T2 without FS } \\
\text { (ii) } b \text { value: } 1000-1200 \text { with black urine } \\
\text { (iii) Thickness: } 6 \mathrm{~mm} / 0.0 \text { spacing or } 5 \mathrm{~mm} / 1.0 \text { spacing }\end{array}$ \\
\hline Ax PWI & $\begin{array}{l}\text { (i) } 3 \mathrm{D} \text { T1 without FS } \\
\text { (ii) Temporal resolution }<15 \mathrm{sec} \\
\text { (iii) Spatial resolution and slice thickness }=3 \mathrm{~mm} / 0.0 \mathrm{sp} \\
\text { (iv) Size of box }>15 \mathrm{~cm} \\
\text { (v) Loc. per slab }>50 \\
\text { (vi) } 4 \text { acquisitions without gadolinium (baseline) } \\
\text { (vii) Reformat good quality of sagittal and coronal reconstruction }+++ \\
\text { (viii) Time duration: } 4 \mathrm{mn}\end{array}$ \\
\hline Ax or sag T1 FS gadolinium & (i) Copy Ax or sag T1FS without gadolinium \\
\hline
\end{tabular}

Others types of solid component such as thin smooth septa or cyst wall are not considered as solid tissue and do not require any functional characterization. If solid tissue is detected within an adnexal lesion, early publications demonstrated that the T2 signal intensity of the solid tissue is useful to distinguish benign from malignant tumors [12] because some benign tumors contain fibrous material in their solid tissue and thus appear with a typically low T2 weighted signal. The accuracy of MRI interpretation to differentiate benign from malignant masses using only the conventional sequences is about $80 \%$ according to the published literature [17, 20-22]. However, Huchon et al. demonstrated that most ovarian tumors undergo surgery without any MR analysis and there is a high rate of incomplete surgery [23]. More recently, perfusion and diffusion weighted sequences were demonstrated to improve diagnostic confidence about $25 \%$ and $15 \%$, respectively [24], allowing an accuracy of up to $94.6 \%$. This increase in the diagnostic accuracy of MRI may help to increase the clinician's confidence in MR imaging.

This review will present these two functional techniques that are readily available in clinical routine, including acquisition parameters, method of analysis, and added value compared with conventional images. Then, we will propose a synthesis consisting of an interpretation model combining conventional and functional criteria.

\section{Perfusion Weighted MR Sequence}

Ovarian cancer is characterized by an anarchic neovascularization resulting in a wide number of immature microvessels. These vessels are characterized by a lack of coverage by pericytes and the higher expression of one of the receptors of
Vascular Endothelial Growth Factor (VEGF) named VEGFR2 on both endothelial and epithelial cells of ovarian cystadenocarcinomas. These physiopathogenic characteristics have been demonstrated to be in line with variations of MR perfusion parameters [25].

2.1. Technical Features. All the parameters required to perform MRI for adnexal masses characterization are provided in Table 1 .

2.2. MR Acquisition Parameters. For female pelvic imaging, perfusion MR technique is based on a Dynamic Contrast Enhanced (DCE) Gradient Echo (GRE) T1 weighted sequence. The main important parameters include a temporal resolution which must be lower than 15 seconds and the total sequence duration should be at least 3 minutes. External myometrium is used as an internal reference because it is enhanced to approximately the same extent as ovarian tumour tissue [26]. Ideally, an axial 3D GRE T1 sequence should be performed with high quality reformats postcontrast in the sagittal and coronal planes. If only a $2 \mathrm{D}$ sequence can be performed, the acquisition plane should be placed in order to cover external myometrium and the tumor, both of which need to be analyzed [27] (Figure 1).

2.3. MR Analysis. For perfusion data, three types of analysis exist. The first one is the time intensity curve analysis. For adnexal mass characterization, as shown in Figure 1, two regions of interest are placed on both external myometrium and solid tissue of the adnexal mass on DCE MR sequence. Then, the evolution of relative signal intensity according to time can be assessed using time intensity curves. For 


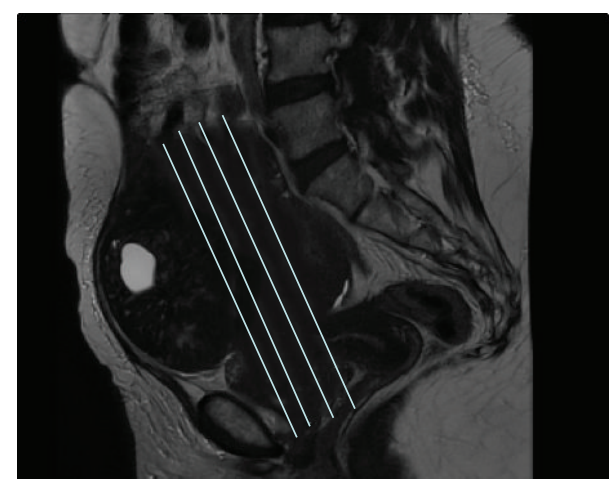

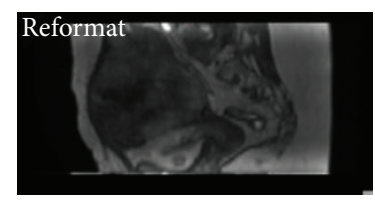

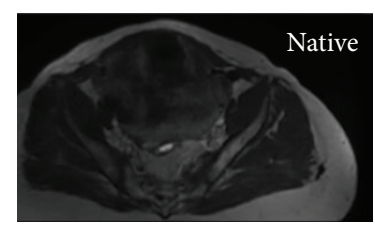

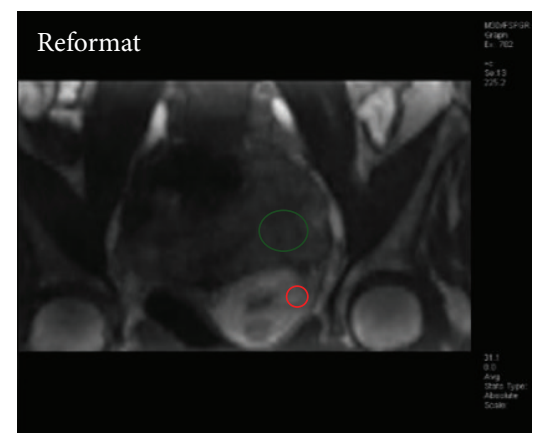

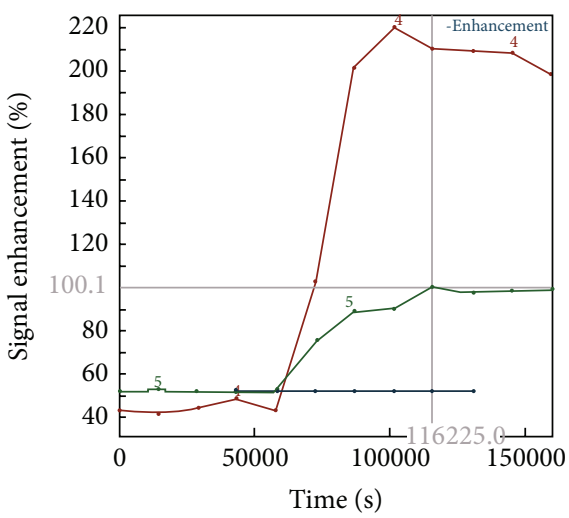

FIgURE 1: Perfusion MR acquisition. The sequence may be acquired in 2D plane in order to cover both external myometrium and the tumor or in axial 3D plane with a high quality of reformatting imaging in sagittal and coronal planes.

characterization of adnexal masses, time intensity curve of solid tissue in an adnexal mass is compared to that of external myometrium, which acts as the internal reference. The use of an internal reference overcomes to some extent the lack of reproducibility which is well known to be the main limitation of this type of postcontrast perfusion data. Thus, when solid tissue is enhanced with a weak and progressive curve in comparison to the myometrium, the curve is named "type 1." When solid tissue is enhanced with a moderate enhancement in comparison to myometrium with a plateau, the curve is named "type 2." Finally, when solid tissue is enhanced with a curve steeper than that of myometrium, whatever the intensity of enhancement, the curve is named "type 3" [28] (Figure 2).

The second type of analysis is named semiquantitative analysis based also on relative signal intensity of the curve as descriptive analysis. Area under the enhancing curve may be easily calculated and the initial area under the curve (before $60 \mathrm{sec}$ ) named Initial Area under Curve (IAUC60) has been demonstrated useful for adnexal masses characterization $[28,29]$. Using different mathematic models, the time intensity curve can be fitted. With a high temporal resolution acquisition data, Thomassin-Naggara et al. determined three semiquantitative parameters by fitting with Hill equation: enhancement amplitude (EA), time of half rising (THR), and maximal slope (MS) of the curve. These parameters were demonstrated to be useful to characterize adnexal masses because a correlation was proven between enhancement amplitude and maximal slope with pericyte coverage index

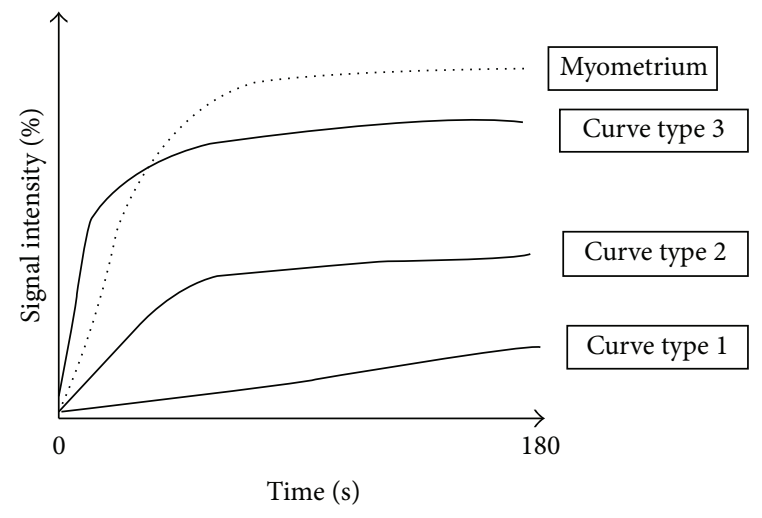

FIgURE 2: Time intensity curve.

(PCI) and high expression of VEGFR-2 on both epithelial and endothelial cells [25]. Moreover, an independent external validation of these parameters was performed later on another population studied in another center [29]. With a lower temporal resolution acquisition data of 30 seconds, Dilks et al. demonstrated the usefulness of other parameters including mean $\mathrm{SI}_{\max }$, a lower $\mathrm{SI}_{\text {rel }}$, and a wash-in rate (WIR) that corresponds to the ratio between enhancement amplitude and time [30] (Figure 3). These authors underlined the simplicity of this technique because the software used to calculate enhancement parameters is widely available on MR imaging workstations [31]. 


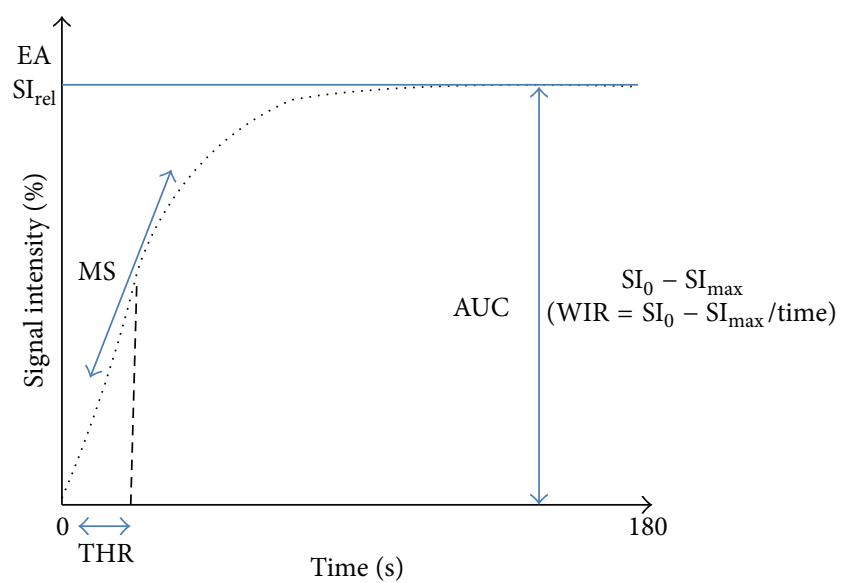

FIGURE 3: Semiquantitative analysis. Different parameters published were EA (enhancement amplitude), THR (time of half rising), maximal slope (MS), $\mathrm{SI}_{\text {rel }}$ (maximal relative enhancement), WIR (wash-in rate), and $\mathrm{SI}_{\max }$.

As we underlined before, descriptive and semiquantitative analysis are based on signal intensity evaluation. However, using MR imaging, signal intensity depends mainly on the type of acquisition parameters, such as flip angle and TR. Image contrast provided by the administration of contrast agent and linearity between signal intensity variation and contrast agent concentration are highly dependent on these parameters [32]. Thus, many authors argue that we need to obtain reproducible perfusion parameters independent of acquisition conditions. We needed to develop perfusion parameters that are not expressed according to signal intensity but to gadolinium concentration [33]. Then, a more recent approach consists in a quantitative analysis which is based on a pharmacokinetic modeling allowing conversion of signal intensity into gadolinium concentration $[34,35]$. Depending on the temporal resolution of the acquisition, different pharmacokinetic models may be applied. At low temporal resolution acquisition $(>10 \mathrm{sec})$, Carter et al. demonstrated the usefulness of Tofts-Kety model to differentiate benign from malignant tumors using $K_{\text {trans }}$ and $K_{\text {ep }}$ [29]. Tofts-Kety model is the most used pharmacokinetic model in this context thanks to its good reproducibility [35]. However, the original Tofts-Kety is not a physiological model resulting in parameters with values depending on acquisition settings [36]. Moreover, descriptive and semiquantitative analysis showed that the initial part of the curve is the most informative to distinguish benign from malignant tumors which suggests that tissue blood flow would be the most informative parameter. Thus, using high temporal resolution acquisition ( $3 \mathrm{sec}$ ) [37], de Bazelaire et al. described a Brix modified model [38] that allows the determination of tissue blood flow $\left(F_{T}\right)$, blood volume fraction $\left(V_{b}\right)$, permeabilitysurface area product $\left(P_{S}\right)$, and interstitial volume fraction $\left(V_{e}\right)$ and this proved useful for adnexal mass characterization [39] (Figure 4).

2.4. Added Value for Adnexal Mass Characterization. The perfusion weighted MR sequence provides additional criteria for adnexal characterization.
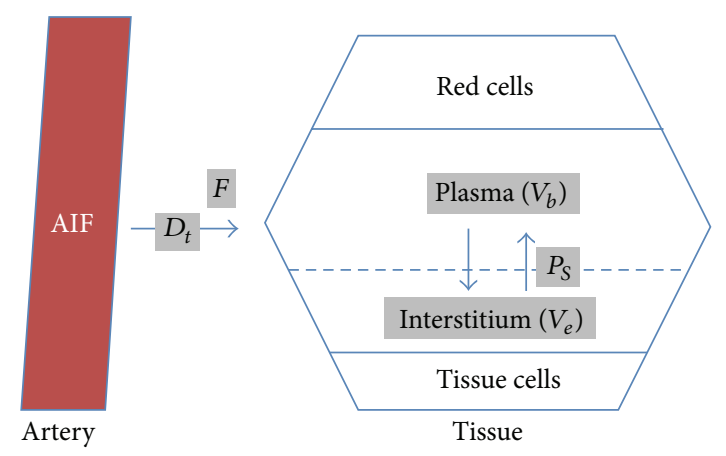

FIGURE 4: Pharmacokinetic model: Brix modified model with 4 quantitative parameters including tissue blood flow $(F)$, blood volumetric fraction $\left(V_{b}\right)$, the product of capillary wall permeability and surface area $\left(P_{S}\right)$, interstitial volume $\left(V_{e}\right)$, and the delay for the contrast media to reach tissue $\left(D_{t}\right)$.

Using descriptive analysis, first publications demonstrated in a population of 37 epithelial tumors that a slow, low-level enhancement pattern typical of a type 1 curve has a sensitivity of $70 \%$ and a specificity of $88.8 \%$ for benign tumor, whereas a rapid and high level of enhancement typical of a type 3 curve was only found in invasive malignant tumors with a sensitivity of $67 \%$. A type 2 curve was mostly found in borderline tumors (sensitivity $72.4 \%$ ) but with a lower specificity [28]. The added value of perfusion weighted imaging was tested in a larger population of 87 complex adnexal masses [24] and the addition of time intensity curve analysis resulted in an increase in diagnostic confidence of $25 \%$ for a senior reader in pelvic MR imaging. The diagnosis was correctly changed in 7\% (3/41) of malignant masses and in $62 \%(10 / 16)$ of benign masses. No diagnosis was incorrectly changed. In our experience, the time intensity curve analysis is particularly interesting for benign tumors with fibrous component such as ovarian fibroma or cystadenofibroma that do not always display a low T2 signal due to oedematous areas. These lesions typically enhance with time intensity curve type 1 [40] (Figure 5). Moreover, time intensity curve analysis is also useful to differentiate borderline from invasive malignant tumors. Typically, borderline tumors display solid papillary projections whereas invasive malignant tumors display a solid mass with or without solid papillary projections. When there is no clear solid mass but grouped solid papillary projection, it can be difficult to be sure if the tumor is only a borderline tumor or an early invasive ovarian cancer. Another application of time intensity curve analysis is to help characterize a solid pelvic mass when the ovarian or uterine origin cannot be clearly identified. In menopausal women, normal ovarian parenchyma is more difficult to identify on T2 weighted sequences than in premenopausal women because follicles are usually no longer seen. The two most frequent solid pelvic masses in women are uterine leiomyoma and ovarian fibroma. When a uterine leiomyoma is pedunculated, morphological criteria are sometimes insufficient to be sure of the origin. Moreover, T2 signal intensity is not very useful to distinguish these two tumors. Time intensity curve analysis can be useful in this situation because uterine leiomyomas are typically enhanced with virtually the same time intensity 

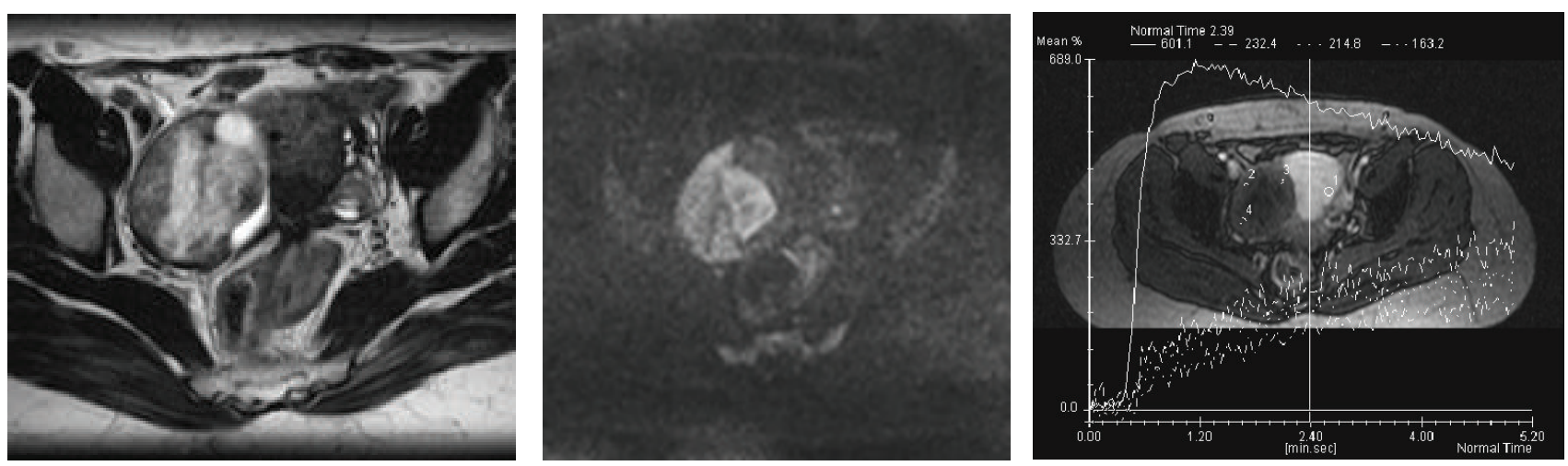

FIgURE 5: Ovarian fibroma right adnexal mixed cystic solid mass with an intermediate T2 weighted signal intensity in the solid component (A), a high DW signal (B), and a time intensity curve weak and progressive without any plateau (dotted line) in comparison with myometrial enhancement (continuous line).
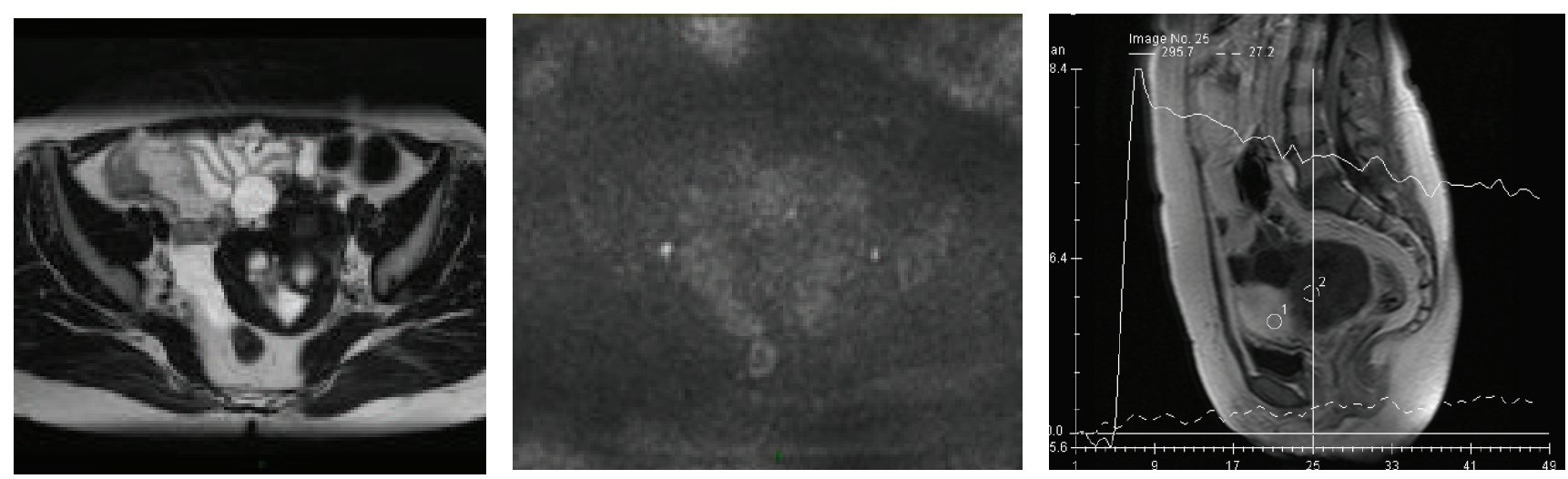

FIGURE 6: T2 "dark though" effect—cystadenofibroma. The fibrous component of this tumor was highly cellular with an ADC value lower than $1 \cdot 10^{-3} \mathrm{~mm}^{2} / \mathrm{s}$. However, the tumor is not bright on DW image because of its low T2 signal (T2 dark through effect).

curve as the myometrium whereas ovarian fibromas display a weak and progressive manner (type 1 curve) [41] (Figure 6).

Using semiquantitative analysis, Bernardin et al. demonstrated that benign lesions displayed a lower mean maximal signal intensity $\left(\mathrm{SI}_{\max }\right)$, a lower relative signal intensity $\left(\mathrm{SI}_{\text {rel }}\right)$, and a lower wash-in rate (WIR) than borderline and malignant tumors [42] (Figure 3). In this study, the authors found a sensitivity of $67 \%$ and specificity of $88 \%$ in predicting borderline/invasive malignancy applying a cutoff WIR of $9.51 / \mathrm{s}$, although there was overlap between borderline and benign lesion with a range of 2-81/s. Moreover, in a more recent study, the same team confirms that all benign cystadenofibroma solid tissues have a WIR less than 5.81/s [40]. Using myometrium as internal reference, Thomassin-Naggara et al. showed that the enhancement amplitude ratio $\left(\mathrm{EA}_{\text {ratio }}\right)$, time of half rising ratio $\left(\mathrm{THR}_{\text {ratio }}\right)$, and maximal slope $\left(\mathrm{MS}_{\text {ratio }}\right)$ were significantly higher in invasive malignant tumors than in benign and borderline tumors. Moreover, in this last study, borderline tumors were characterized by a higher $\mathrm{EA}_{\text {ratio }}$ than benign tumors whereas no differences between benign and borderline tumors were found using WIR values [43]. Thus, in our experience, the use of myometrium as internal reference improves the diagnostic value of semiquantitative parameters when viewed in combination with the descriptive parameters.

Using quantitative analysis, Thomassin-Naggara et al. demonstrated that benign tumors displayed a lower tissue blood flow $\left(F_{T}\right)$, a lower blood fraction volume $\left(V_{b}\right)$, a higher interstitial volume $\left(V_{e}\right)$, and a lower relative AUC (rAUC) than malignant tumors. Moreover, borderline tumors displayed a lower $F_{T}$ and a higher $D_{t}$ than invasive ovarian tumors [39]. With data acquired at lower temporal resolution, malignant tumors display a higher $K_{\text {ep }}$ in their solid component than benign tumors $(P<0,001)[29]$.

\section{Diffusion Weighted MR Sequence}

Diffusion weighted (DW) MR imaging is based on the analysis of the movement of water molecules in a tissue. In a highly cellular tissue, a restriction of the movement of water molecules (a reduction in diffusivity) exists between cells and the tissue appears as high signal intensity on high $b$ value DW image with associated low ADC (apparent diffusion coefficient). In less cellular tissue, there is less restriction of the movement of water molecules. Thus, ADC is high 
and the tissue typically appears without any high signal intensity on the high b value DW images. Indeed, depending on acquisition parameters, DW signal will be due to both cellularity (ADC values) and T2 signal.

3.1. MR Acquisition Parameters and MR Analysis. For adnexal mass characterization, many studies have underlined the usefulness of DW signal intensity, although the ADC analysis for solid component analysis can be unhelpful [29, 44, 45]. In fact, many benign tumors have fibrous tissue that restricts the movement of water molecules. Thus, there is a great overlap between ADC values of the solid tissue of benign and malignant tumors [29, 45]. However, as the DW signal is the combination of water diffusivity and T2 signal intensity, the typically very low T2 weighted signal intensity of fibrous tissue in these benign adnexal tumors decreases the theoretical high DW signal intensity due to the high cellularity of these tumors (T2 dark though effect) (Figure 6). Thus, the analysis of DW signal allows correct classification as benign in this group of tumors and makes such criteria accurate to distinguish benign from malignant adnexal tumors.

Then, as mainly DW signal is useful for adnexal mass characterization, we need to optimize a sequence to obtain a sequence with the lowest T2 signal effect with a maximal contrast to noise ratio. T2 signal effect decreases with the increase of $b$ values. At $b_{0}$ value, the sequence is weighted as a T2 weighted sequence. To find the optimal $b$ value, we use urine as internal reference. As we want to suppress T2 effect, we need to obtain dark urine. In our experience, the right $b$ value is between $b_{1000}$ and $b_{1200}$ depending on the manufacturer.

3.2. Added Value for Adnexal Masses Characterization. Many publications have studied the value of diffusion MR sequence for adnexal mass characterization. First publications studied the value of diffusion weighted MR imaging to characterize endometriotic cystic component or mature cystic teratoma [46]. The accuracy of MR imaging with conventional sequences is very high for these benign masses (up to 95\%) [14, 47-49]. Thus, the added value of diffusion weighted imaging in this setting is limited and has never been statistically demonstrated. Nevertheless, in mature cystic teratoma with paucity of fat, the very low ADC value should be useful to identify keratinoid content $[46,50]$.

Using DWI to characterize solid tissue, many authors demonstrate that ADC values are not useful to distinguish benign from malignant tumors due to the overlap with benign fibrous tumors $[44,51]$. However, thanks to "dark through" effect, the value of the absence of DW signal was demonstrated for predicting benignity (positive likelihood ratio $=10.1)$. Moreover, the combination of a low T2 weighted signal with a low DW signal allows a confident exclusion of malignancy. These preliminary data were confirmed in further studies that underlined the added value of diffusion weighted sequence to increase diagnostic confidence in $15 \%$ and especially for benign tumors [24]. The diagnosis was correctly changed in $8.9 \%$ (4/45) of malignant masses and in $28 \%(8 / 28)$ of benign masses. One diagnosis was incorrectly changed in one case of ovarian fibroma (Figure 7).

\section{Combination of Functional Parameters in an Interpretation Model}

Finally, some studies have evaluated the usefulness of the added combination of perfusion and diffusion weighted analysis to conventional analysis. These studies demonstrated that functional analysis is useful especially for reclassifying as benign tumors that were misclassified as malignant using conventional criteria. In our experience, that is the main issue for clinical practice. Indeed, recent trials have shown that unnecessary interventions in women with benign adnexal lesions lead to significant morbidity and mortality [52]. All women have operative and anesthetic risks, which are increased in menopausal women with additional comorbidity factors (obesity, diabetes, and hypertension). Moreover, in premenopausal women, preservation of fertility is a major issue as ovarian surgery mainly based on cystectomy is associated with the risk of ovarian reserve alteration, especially for cysts greater than $5 \mathrm{~cm}$ in diameter $[53,54]$.

Although MRI is the most accurate imaging technique to characterize adnexal masses [54, 55], a debate exists on its use among clinicians explaining why only $25 \%$ of surgeons performed MR imaging before surgery and thus the high incidence of preoperative misdiagnoses [23].

One hypothesis is the lack of standardization of the MR report and the absence of combination of conventional and functional criteria and this could in part be responsible. In this context, the first MR diagnostic score was recently built and described each complex adnexal mass according its positive predictive value of malignancy with a five categories score named $A_{\text {DNEX }} M_{\text {SCORING }}$ system [19] (Table 2). If the complex mass does not display any enhancing solid tissue, the classification is $\mathrm{A}_{\mathrm{DNEX}} \mathrm{MR}_{\mathrm{SCORE}} 2$ or 3 with a PPV lower than $5 \%$. If a complex adnexal mass displays an enhancing solid tissue, in the absence of peritoneal implants, we need to take account of the evaluation of diffusion weighted (DW) signal and time intensity curve analysis. Functional sequences are included in this score as follows: If there is a low T2 and low DW signal on the high $b$ value image, the tumor is always benign $\left(A_{D N E X} M R_{S C O R E} 2\right)$. If not, time intensity curve analysis allows differentiating probably benign masses ( $A_{\text {DNEX }} M_{\text {SCORE }} 3$ ) (if the solid tissue is enhanced according to a type 1 time intensity curve), indeterminate mass $\left(\mathrm{A}_{\mathrm{DNEX}} \mathrm{MR}_{\text {SCORE }} 4\right)$ (if the solid tissue is enhanced according to a type 2 time intensity), and probably malignant mass ( $\mathrm{A}_{\mathrm{DNEX}} \mathrm{MR}_{\mathrm{SCORE}}$ ) (if the solid tissue is enhanced with a type 3 time intensity curve) (Figure 8). This score was developed and validated with a very high accuracy (AUROC > 0.94). A score $\leq 3$ has been associated with benignity with a sensitivity of $96.6 \%$ and a specificity of $93.5 \%$. A multicentre European prospective study (the "EURAD study") is currently in recruitment, with the aim of validating the proposed scoring system (clinical trial NCT01738789). The potential impact of 
TABLE 2: $\mathrm{A}_{\text {DNEX }} \mathrm{MR}_{\text {SCORING }}$ system.

\begin{tabular}{|c|c|c|}
\hline $\mathrm{A}_{\mathrm{DNEX}} \mathrm{MR}_{\mathrm{SCORE}} 1$ & Absence of mass & - \\
\hline $\mathrm{A}_{\mathrm{DNEX}} \mathrm{MR}_{\mathrm{SCORE}} 2$ & Benign mass & $\begin{array}{l}\text { Purely cystic mass } \\
\text { Purely endometriotic mass } \\
\text { Purely fatty mass } \\
\text { Mass without wall enhancement } \\
\text { Low T2 and low DW signal of solid component }\end{array}$ \\
\hline $\mathrm{A}_{\mathrm{DNEX}} \mathrm{MR}_{\mathrm{SCORE}} 3$ & Probably benign mass & $\begin{array}{l}\text { Bi- or multiloculate cyst without solid component } \\
\text { Curve type } 1 \text { of solid component }\end{array}$ \\
\hline $\mathrm{A}_{\mathrm{DNEX}} \mathrm{MR}_{\mathrm{SCORE}} 4$ & Indeterminate & All others lesions (including curve type 2 of solid component) \\
\hline $\mathrm{A}_{\mathrm{DNEX}} \mathrm{MR}_{\mathrm{SCORE}} 5$ & Probably malignant mass & $\begin{array}{l}\text { Curve type } 3 \text { of solid component } \\
\text { Peritoneal implants }\end{array}$ \\
\hline
\end{tabular}

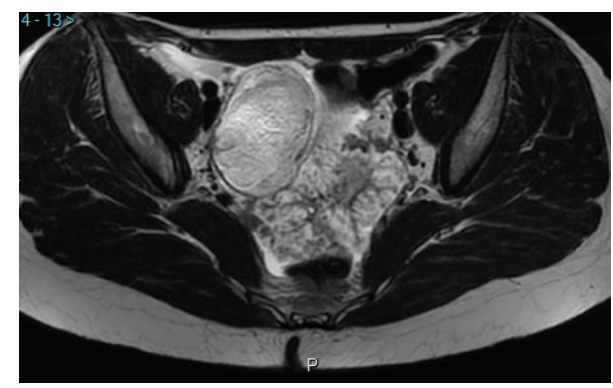

(a)

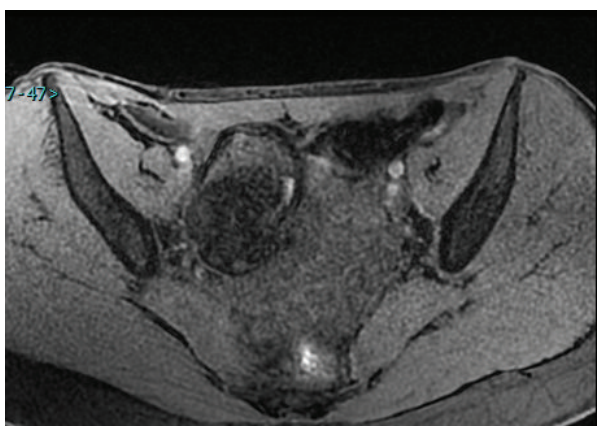

(c)

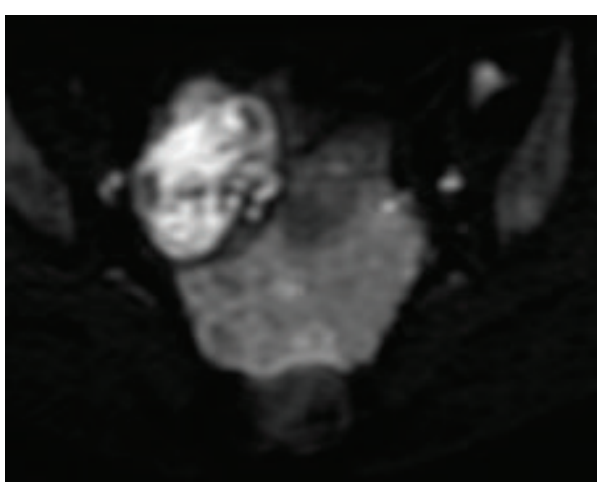

(e)

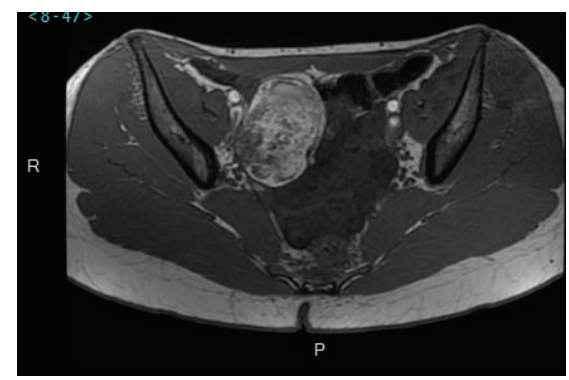

(b)

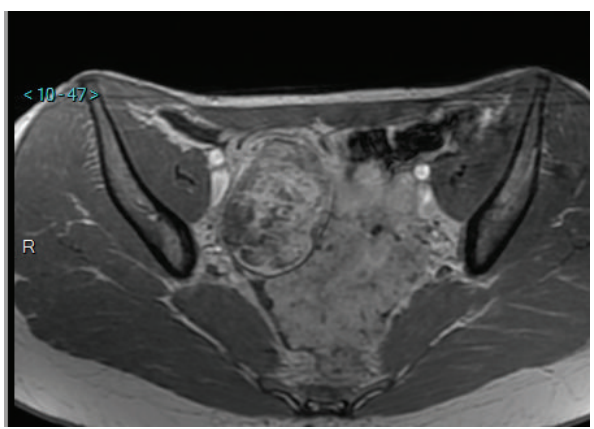

(d)

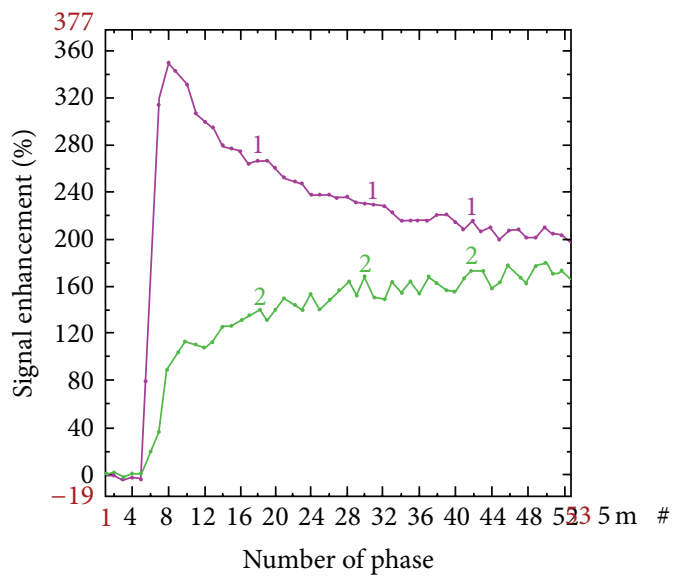

(f)

FIGURE 7: Added value of perfusion and diffusion weighted imaging. Right side: mature cystic teratoma (purely fatty mass: $A_{D N E X} M_{\text {score }}$ 2). Left side: borderline serous cystadenoma (solid tissue which is bright on T2 and DW sequence and that enhances with a TIC type 2: $A_{\text {DNEX }} M_{\text {score }}$ 4). T2 weighted sequence (a), T1 weighted sequence (b), T1 weighted sequence with fat saturation (c), T1 weighted sequence with gadolinium (d), DW sequence (e), and PW analysis comparing myometrial TIC (1) and tumoral TIC (2) (f). 


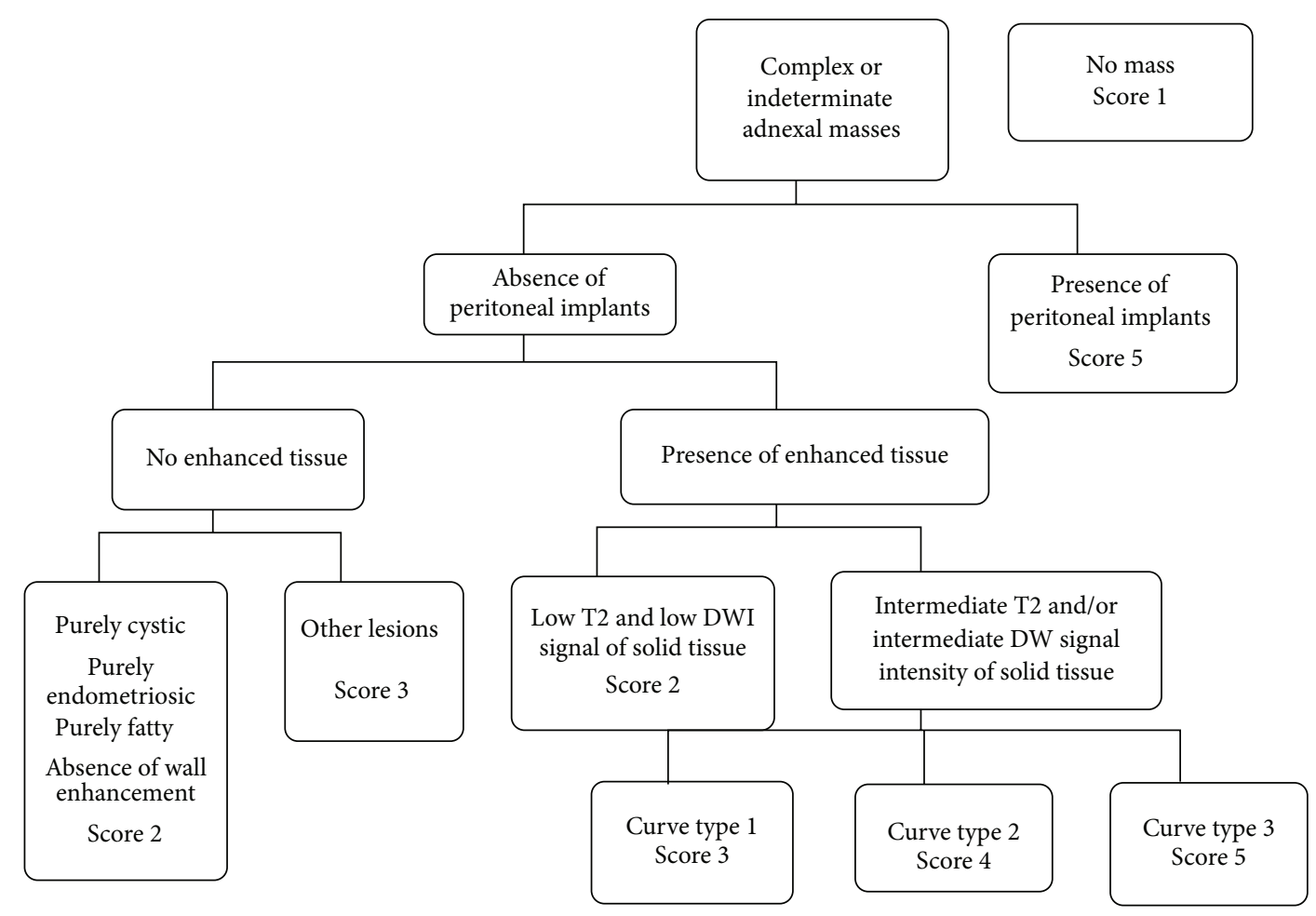

Figure 8: $A_{\text {DNEX }}$ MR scoring system (19).

the score on therapeutic management will then be tested in a future trial.

In this review, we present the development of perfusion and diffusion analysis to characterize sonographically indeterminate adnexal masses. Using time intensity curve analysis and visual assessment of DW signal, functional criteria help the radiologist to improve lesion characterization especially for benign lesions and should help the clinician to avoid unnecessary surgeries. Currently, few data are available to validate in clinical routine more reproducible perfusion analysis for the characterization of adnexal masses. Very recently, new developments of DWI were reported thanks to $3 \mathrm{~T}$ acquisition including the analysis of the heterogeneity of the tumor (ADC entropy) [51]. This method of research would be interesting in the future to find new criteria of characterization.

\section{Key Points}

(1) Perfusion weighted and diffusion weighted MR imaging help to characterize adnexal masses.

(2) Benign tumors with solid components typically display a low DWI signal.

(3) Benign solid tumors are typically enhanced with a type 1 time intensity curve.

(4) $A_{\text {DNEX }} M_{\text {SCORING }}$ system is helpful to relay the radiologist's suspicion of malignancy.

\author{
Abbreviations \\ MR: $\quad$ Magnetic resonance \\ VEGF: Vascular Endothelial Growth Factor \\ DCE: Dynamic Contrast Enhanced \\ GRE: Gradient Echo \\ IAUC: Initial Area under Curve \\ EA: $\quad$ Enhancement amplitude \\ THR: Time of half rising \\ MS: $\quad$ Maximal slope \\ PCI: Pericyte coverage index \\ WIR: Wash-in rate \\ $F_{T}$ : Tissue blood flow \\ $V_{b}: \quad$ Blood volume fraction \\ $P_{S}$ : $\quad$ Permeability-surface area product \\ $V_{e}: \quad$ Interstitial volume fraction \\ TR: Time of relaxation \\ $\mathrm{SI}_{\max }$ : Maximum signal intensity \\ EA $_{\text {ratio: }}$ Enhancement amplitude ratio \\ $\mathrm{THR}_{\text {ratio }}$ : Time of half rising ratio \\ $\mathrm{MS}_{\text {ratio }}$ : Maximal slope \\ ADC: Apparent diffusion coefficient.
}

\section{Disclosure}

As this paper is a review, no ethics committee approval is required. I. Thomassin-Naggara (Invited conference for General Electric, Consultant for Olea). A. Rockall (Invited conference for Guerbet). 


\section{Conflict of Interests}

The authors declare that there is no conflict of interests regarding the publication of this paper.

\section{References}

[1] M. Emoto, H. Iwasaki, K. Mimura, T. Kawarabayashi, and M. Kikuchi, "Differences in the angiogenesis of benign and malignant ovarian tumors, demonstrated by analyses of color Doppler ultrasound, immunohistochemistry, and microvessel density," Cancer, vol. 80, no. 5, pp. 899-907, 1997.

[2] E. M. Schutter, P. Kenemans, and C. Sohn, "Diagnostic value of pelvic examination, ultrasound, and serum CA 125 in postmenopausal women with a pelvic mass. An international multicenter study," Cancer, vol. 74, no. 4, pp. 1398-1406, 1994.

[3] M. H. Vuento, J. P. Pirhonen, J. I. Makinen, P. J. Laippala, M. Gronroos, and T. A. Salmi, "Evaluation of ovarian findings in asymptomatic postmenopausal women with color doppler ultrasound," Cancer, vol. 76, no. 7, pp. 1214-1218, 1995.

[4] D. L. Brown, M. C. Frates, F. C. Laing et al., "Ovarian masses: can benign and malignant lesions be differentiated with color and pulsed Doppler US?” Radiology, vol. 190, no. 2, pp. 333-336, 1994.

[5] D. Levine, D. L. Brown, R. F. Andreotti et al., "Management of asymptomatic ovarian and other adnexal cysts imaged at US: society of radiologists in ultrasound consensus conference statement," Radiology, vol. 256, no. 3, pp. 943-954, 2010.

[6] D. Timmerman, A. C. Testa, T. Bourne et al., "Logistic regression model to distinguish between the benign and malignant adnexal mass before surgery: a multicenter study by the International Ovarian Tumor Analysis Group," Journal of Clinical Oncology, vol. 23, no. 34, pp. 8794-8801, 2005.

[7] K. Kinkel, H. Hricak, Y. Lu, K. Tsuda, and R. A. Filly, "US characterization of ovarian masses: a meta-analysis," Radiology, vol. 217, no. 3, pp. 803-811, 2000.

[8] Y.-Y. Jeong, E. K. Outwater, and H. K. Kang, "Imaging evaluation of ovarian masses," Radiographics, vol. 20, no. 5, pp. 14451470, 2000.

[9] J. W. Walsh, K. J. W. Taylor, J. F. Wasson, P. E. Schwartz, and A. T. Rosenfield, "Gray-scale ultrasound in 204 proved gynecologic masses: accuracy and specific diagnostic criteria," Radiology, vol. 130, no. 2, pp. 391-397, 1979.

[10] M. Bazot, E. Daraï, D. J. Biau, M. Ballester, and L. Dessolle, "Learning curve of transvaginal ultrasound for the diagnosis of endometriomas assessed by the cumulative summation test (LC-CUSUM)," Fertility and Sterility, vol. 95, no. 1, pp. 301-303, 2011.

[11] J. Yazbek, S. K. Raju, J. Ben-Nagi, T. K. Holland, K. Hillaby, and D. Jurkovic, "Effect of quality of gynaecological ultrasonography on management of patients with suspected ovarian cancer: a randomised controlled trial," The Lancet Oncology, vol. 9, no. 2, pp. 124-131, 2008.

[12] E. S. Siegelman and E. K. Outwater, "Tissue characterization in the female pelvis by means of MR imaging," Radiology, vol. 212, no. 1, pp. 5-18, 1999.

[13] K. Kinkel, Y. Lu, A. Mehdizade, M.-F. Pelte, and H. Hricak, "Indeterminate ovarian mass at US: incremental value of second imaging test for characterization-meta-analysis and Bayesian analysis," Radiology, vol. 236, no. 1, pp. 85-94, 2005.
[14] M. Bazot, E. Darai, R. Hourani et al., "Deep pelvic endometriosis: MR imaging for diagnosis and prediction of extension of disease," Radiology, vol. 232, no. 2, pp. 379-389, 2004.

[15] K. A. Jain, D. L. Friedman, T. W. Pettinger, R. Alagappan, R. B. Jeffrey Jr., and F. G. Sommer, "Adnexal masses: comparison of specificity of endovaginal US and pelvic MR imaging," Radiology, vol. 186, no. 3, pp. 697-704, 1993.

[16] T. Komatsu, I. Konishi, M. Mandai et al., "Adnexal masses: transvaginal US and gadolinium-enhanced MR imaging assessment of intratumoral structure," Radiology, vol. 198, no. 1, pp. 109-115, 1996.

[17] Y. Yamashita, M. Torashima, Y. Hatanaka et al., "Adnexal masses: accuracy of characterization with transvaginal US and precontrast and postcontrast MR imaging," Radiology, vol. 194, no. 2, pp. 557-565, 1995.

[18] D. Timmerman, L. Valentin, T. H. Bourne, W. P. Collins, H. Verrelst, and I. Vergote, “Terms, definitions and measurements to describe the sonographic features of adnexal tumors: a consensus opinion from the International Ovarian Tumor Analysis (IOTA) group," Ultrasound in Obstetrics \& Gynecology, vol. 16, no. 5 , pp. 500-505, 2000.

[19] I. Thomassin-Naggara, E. Aubert, A. Rockall et al., "Adnexal masses: development and preliminary validation of an MR imaging scoring system," Radiology, vol. 267, no. 2, pp. 432-443, 2013.

[20] M. Bazot, D. Haouy, E. Daraï, A. Cortez, S. Dechoux-Vodovar, and I. Thomassin-Naggara, "Is MRI a useful tool to distinguish between serous and mucinous borderline ovarian tumours?" Clinical Radiology, vol. 68, no. 1, pp. e1-e8, 2013.

[21] M. Bazot, J. Nassar-Slaba, I. Thomassin-Naggara, A. Cortez, S. Uzan, and E. Darai, "MR imaging compared with intraoperative frozen-section examination for the diagnosis of adnexal tumors; correlation with final histology," European Radiology, vol. 16, no. 12, pp. 2687-2699, 2006.

[22] S. A. Sohaib, A. Sahdev, P. van Trappen, I. J. Jacobs, and R. H. Reznek, "Characterization of adnexal mass lesions on MR imaging," American Journal of Roentgenology, vol. 180, no. 5, pp. 1297-1304, 2003.

[23] C. Huchon, A.-S. Bats, C. Bensaïd et al., "Adnexal masses management: a prospective multicentric observational study," Gynecologie Obstetrique Fertilite, vol. 36, no. 11, pp. 1084-1090, 2008.

[24] I. Thomassin-Naggara, I. Toussaint, N. Perrot et al., "Characterization of complex adnexal masses: value of adding perfusionand diffusion-weighted MR imaging to conventional MR imaging," Radiology, vol. 258, no. 3, pp. 793-803, 2011.

[25] I. Thomassin-Naggara, M. Bazot, E. Daraï, P. Callard, J. Thomassin, and C. A. Cuenod, "Epithelial ovarian tumors: value of dynamic contrast-enhanced MR imaging and correlation with tumor angiogenesis," Radiology, vol. 248, no. 1, pp. 148-159, 2008.

[26] I. Thomassin-Naggara, D. Balvay, C. A. Cuenod, E. Daraï, C. Marsault, and M. Bazot, "Dynamic contrast-enhanced MR imaging to assess physiologic variations of myometrial perfusion," European Radiology, vol. 20, no. 4, pp. 984-994, 2010.

[27] I. Thomassin-Naggara, C. A. Cuenod, E. Darai, C. Marsault, and M. Bazot, "Dynamic contrast-enhanced MR imaging of ovarian neoplasms: current status and future perspectives," Magnetic Resonance Imaging Clinics of North America, vol. 16, no. 4, pp. 661-672, 2008. 
[28] I. Thomassin-Naggara, E. Daraï, C. A. Cuenod, R. Rouzier, P. Callard, and M. Bazot, "Dynamic contrast-enhanced magnetic resonance imaging: a useful tool for characterizing ovarian epithelial tumors," Journal of Magnetic Resonance Imaging, vol. 28, no. 1, pp. 111-120, 2008.

[29] J. S. Carter, J. S. Koopmeiners, J. E. Kuehn-Hajder et al., "Quantitative multiparametric MRI of ovarian cancer," Journal of Magnetic Resonance Imaging, vol. 38, no. 6, pp. 1501-1509, 2013.

[30] P. Dilks, P. Narayanan, R. Reznek, A. Sahdev, and A. Rockall, "Can quantitative dynamic contrastenhanced MRI independently characterize an ovarian mass?" European Radiology, vol. 20, no. 9, pp. 2176-2183, 2010.

[31] P. Mohaghegh and A. G. Rockall, "Imaging strategy for early ovarian cancer: characterization of adnexal masses with conventional and advanced imaging techniques," Radiographics, vol. 32, no. 6, pp. 1751-1773, 2012.

[32] M. K. Ivancevic, I. Zimine, F. Lazeyras, D. Foxall, and J.P. Valle, "FAST sequences optimization for contrast media pharmacokinetic quantification in tissue," Journal of Magnetic Resonance Imaging, vol. 14, no. 6, pp. 771-778, 2001.

[33] S. M. Galbraith, M. A. Lodge, N. J. Taylor et al., "Reproducibility of dynamic contrast-enhanced MRI in human muscle and tumours: comparison of quantitative and semi-quantitative analysis," NMR in Biomedicine, vol. 15, no. 2, pp. 132-142, 2002.

[34] K. Hittmair, G. Gomiscek, K. Langenberger, M. Recht, H. Imhof, and J. Kramer, "Method for the quantitative assessment of contrast agent uptake in dynamic contrast-enhanced MRI," Magnetic Resonance in Medicine, vol. 31, no. 5, pp. 567-571, 1994.

[35] P. S. Tofts, "Modeling tracer kinetics in dynamic Gd-DTPA MR imaging," Journal of Magnetic Resonance Imaging, vol. 7, no. 1, pp. 91-101, 1997.

[36] C. A. Cuenod, L. Fournier, D. Balvay, and J.-M. Guinebretière, "Tumor angiogenesis: pathophysiology and implications for contrast-enhanced MRI and CT assessment," Abdominal Imaging, vol. 31, no. 2, pp. 188-193, 2006.

[37] J. L. Evelhoch, "Key factors in the acquisition of contrast kinetic data for oncology," Journal of Magnetic Resonance Imaging, vol. 10, no. 3, pp. 254-259, 1999.

[38] C. de Bazelaire, N. Siauve, L. Fournier et al., "Comprehensive model for simultaneous MRI determination of perfusion and permeability using a blood-pool agent in rats rhabdomyosarcoma," European Radiology, vol. 15, no. 12, pp. 2497-2505, 2005.

[39] I. Thomassin-Naggara, D. Balvay, E. Aubert et al., "Quantitative dynamic contrast-enhanced MR imaging analysis of complex adnexal masses: a preliminary study," European Radiology, vol. 22, no. 4, pp. 738-745, 2012.

[40] Y. Z. Tang, S. Liyanage, P. Narayanan et al., “The MRI features of histologically proven ovarian cystadenofibromas-an assessment of the morphological and enhancement patterns," European Radiology, vol. 23, no. 1, pp. 48-56, 2013.

[41] I. Thomassin-Naggara, E. Daraï, J. Nassar-Slaba, A. Cortez, C. Marsault, and M. Bazot, "Value of dynamic enhanced magnetic resonance imaging for distinguishing between ovarian fibroma and subserous uterine leiomyoma," Journal of Computer Assisted Tomography, vol. 31, no. 2, pp. 236-242, 2007.

[42] L. Bernardin, P. Dilks, S. Liyanage, M. E. Miquel, A. Sahdev, and A. Rockall, "Effectiveness of semi-quantitative multiphase dynamic contrast-enhanced MRI as a predictor of malignancy in complex adnexal masses: radiological and pathological correlation," European Radiology, vol. 22, no. 4, pp. 880-890, 2012.
[43] I. Thomassin-Naggara, E. Darai, C. A. Cuenod, R. Rouzier, P. Callard, and M. Bazot, "Dynamic contrast-enhanced magnetic resonance imaging: a useful tool for characterizing ovarian epithelial tumors," Journal of Magnetic Resonance Imaging, vol. 28, no. 1, pp. 111-120, 2008.

[44] S. Fujii, S. Kakite, K. Nishihara et al., "Diagnostic accuracy of diffusion-weighted imaging in differentiating benign from malignant ovarian lesions," Journal of Magnetic Resonance Imaging, vol. 28, no. 5, pp. 1149-1156, 2008.

[45] I. Thomassin-Naggara, E. Daraï, C. A. Cuenod et al., "Contribution of diffusion-weighted MR imaging for predicting benignity of complex adnexal masses," European Radiology, vol. 19, no. 6, pp. 1544-1552, 2009.

[46] T. Nakayama, K. Yoshimitsu, H. Irie et al., "Diffusion-weighted echo-planar MR imaging and ADC mapping in the differential diagnosis of ovarian cystic masses: usefulness of detecting keratinoid substances in mature cystic teratomas," Journal of Magnetic Resonance Imaging, vol. 22, no. 2, pp. 271-278, 2005.

[47] M. Bazot, F. Boudghéne, P. Billiéres, J.-M. Antoine, S. Uzan, and J.-M. Bigot, "Value of fat-suppression gradient-echo MR imaging in the diagnosis of ovarian cystic teratomas," Clinical Imaging, vol. 24, no. 3, pp. 146-153, 2000.

[48] C. Guinet, J.-N. Buy, M. A. Ghossain et al., "Fat suppression techniques in MR imaging of mature ovarian teratomas: comparison with CT,' European Journal of Radiology, vol. 17, no. 2, pp. 117-121, 1993.

[49] C. Guinet, M. A. Ghossain, J.-N. Buy et al., "Mature cystic teratomas of the ovary: CT and MR findings," European Journal of Radiology, vol. 20, no. 2, pp. 137-143, 1995.

[50] B. Chilla, N. Hauser, G. Singer, M. Trippel, J. M. Froehlich, and R. A. Kubik-Huch, "Indeterminate adnexal masses at ultrasound: effect of MRI imaging findings on diagnostic thinking and therapeutic decisions," European Radiology, vol. 21, no. 6, pp. 1301-1310, 2011.

[51] A. S. Kierans, G. L. Bennett, T. C. Mussi et al., "Characterization of malignancy of adnexal lesions using ADC entropy: comparison with mean ADC and qualitative DWI assessment," Journal of Magnetic Resonance Imaging, vol. 37, no. 1, pp. 164-171, 2013.

[52] S. S. Buys, E. Partridge, M. H. Greene et al., "Ovarian cancer screening in the Prostate, Lung, Colorectal and Ovarian (PLCO) cancer screening trial: findings from the initial screen of a randomized trial," American Journal of Obstetrics \& Gynecology, vol. 193, no. 5, pp. 1630-1639, 2005.

[53] H. Roman, M. Auber, C. Mokdad et al., "Ovarian endometrioma ablation using plasma energy versus cystectomy: a step toward better preservation of the ovarian parenchyma in women wishing to conceive," Fertility and Sterility, vol. 96, no. 6, pp. 1396-1400, 2011.

[54] H. Hricak, M. Chen, F. V. Coakley et al., "Complex adnexal masses: detection and characterization with MR imagingmultivariate analysis," Radiology, vol. 214, no. 1, pp. 39-46, 2000.

[55] E. K. Outwater and C. J. Dunton, "Imaging of the ovary and adnexa: clinical issues and applications of MR imaging," Radiology, vol. 194, no. 1, pp. 1-18, 1995. 


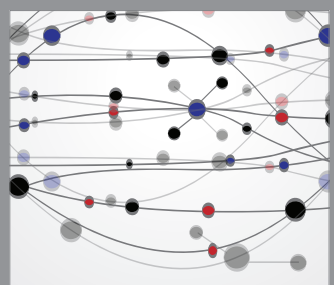

The Scientific World Journal
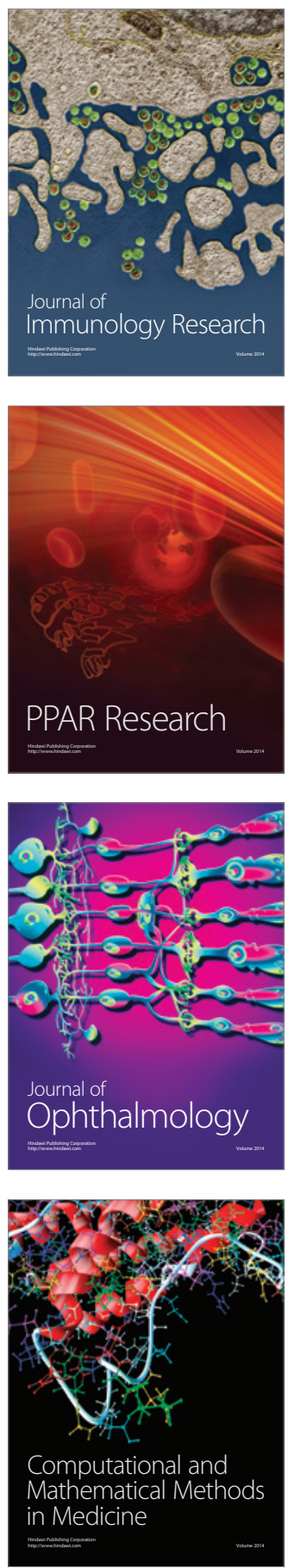

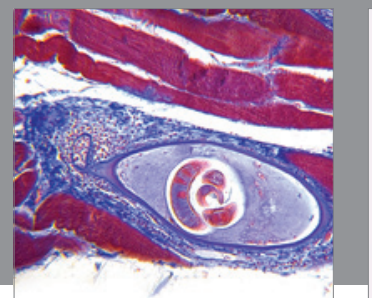

Gastroenterology

Research and Practice
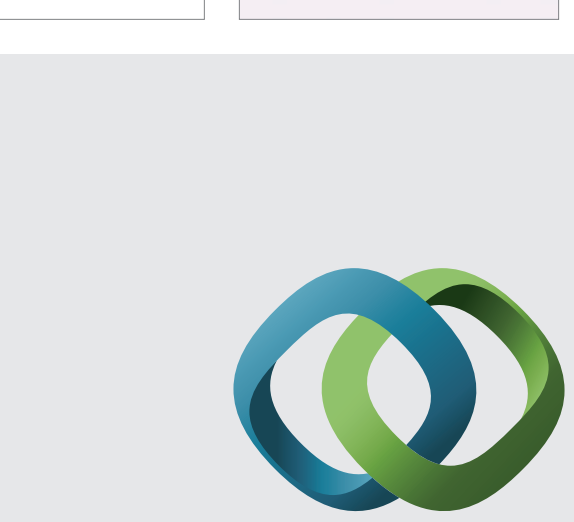

\section{Hindawi}

Submit your manuscripts at

http://www.hindawi.com
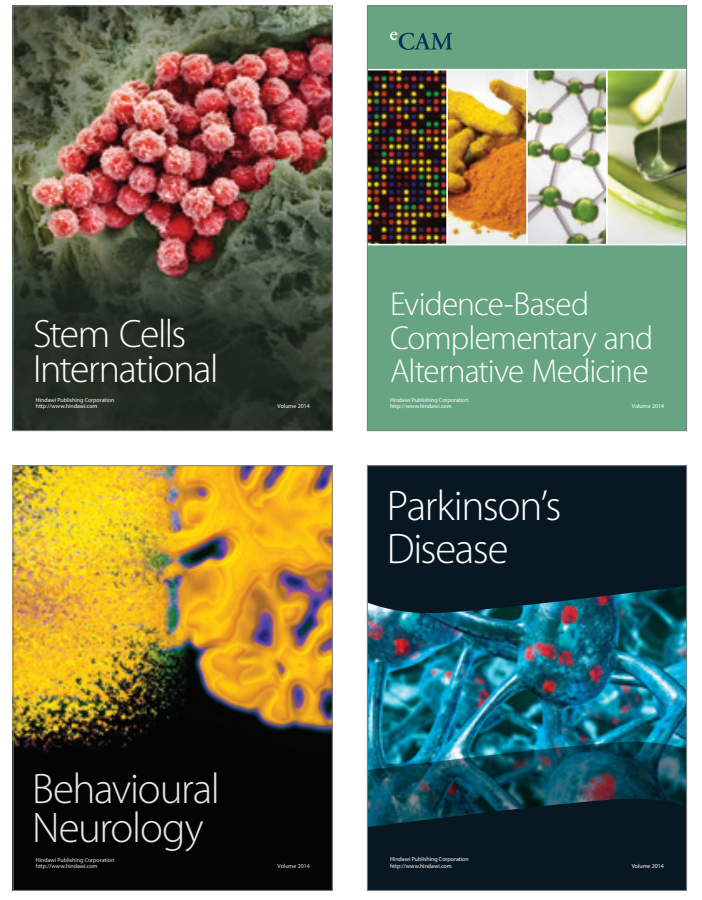
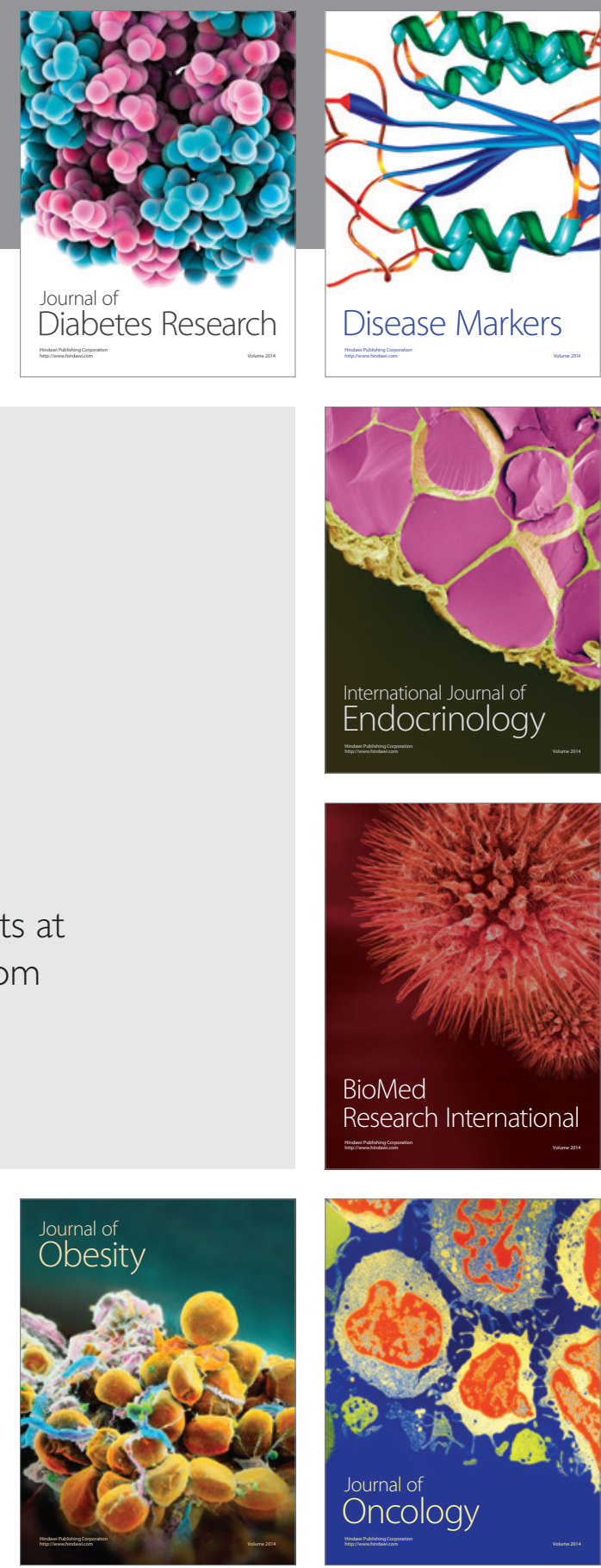

Disease Markers
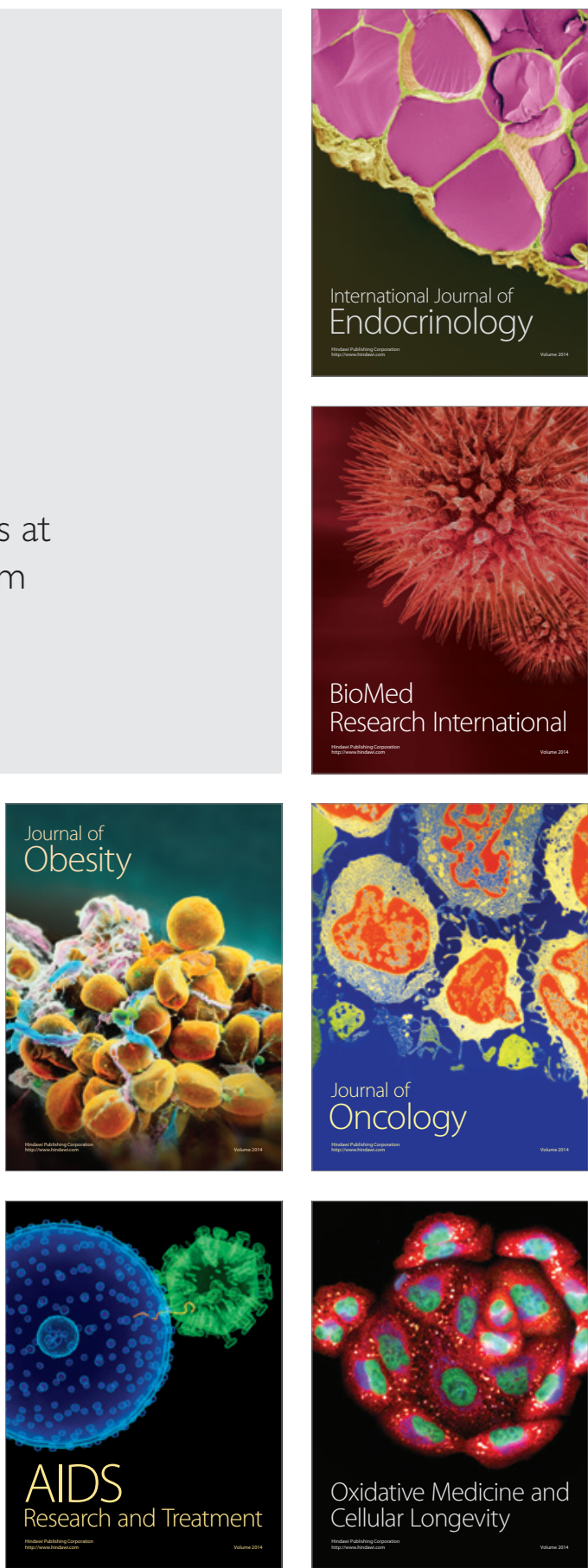